\title{
TRIAGEM DE SAÚDE PARA PARTICIPAÇÃO NOS PROGRAMAS DE EXERCÍCIO FÍSICO PÓS-PANDEMIA DE COVID-19: UMA AÇÃO NECESSÁRIA E EMERGENTE AO PROFISSIONAL DE EDUCAÇÃO FÍSICA
}

\author{
Erivelton Fernandes França ${ }^{1,2}$ \\ Michel Monteiro Macedo \\ Tania Regina de França ${ }^{1}$ \\ Douglas Figueiredo Cossote ${ }^{1}$ \\ Leandro Gonçalves' \\ Paulo Adriano Campos dos Santos ${ }^{3}$ \\ Gabrielle Mitico Miyake ${ }^{4}$
}

As políticas de isolamento social, adotadas pelos governos e autoridades sanitárias em vários países do mundo, se justificam no intuito de atenuar o número de mortes frente à pandemia de COVID-19. Tais condutas são necessárias e contribuem para que não ocorra um colapso dos sistemas públicos de saúde. Em outras palavras, se tais medidas não forem mantidas durante a pandemia, o número de mortes por esta doença poderá crescer devido ao aumento da transmissão viral, ausência de leitos, número insuficiente de profissionais da saúde durante o tratamento, e a ausência de aparatos tecnológicos e insumos hospitalares, necessários para o tratamento eficaz da doença. Dentre esses, podemos citar os respiradores mecânicos e até mesmo os Equipamentos de Proteção Individual (EPI's) como as máscaras de proteção, que durante esta pandemia se tornaram objetos de interesse político/econômico.

Apesar da inquestionável necessidade de políticas de isolamento social nesta pandemia de COVID-19, inevitavelmente ocorre uma diminuição drástica e considerável do nível de atividade física populacional, em todos os grupos etários, inclusive em adultos jovens [1]. Isso ocorre pelo fato de muitos indivíduos passarem a trabalhar em modalidade home office, além de diversos espaços públicos reservados para a prática de atividades físicas e lazer estarem fechados. Outro fator que contribui para a inatividade física durante a pandemia é o receio da população em realizar atividades físicas ao ar livre, como caminhada e corrida, devido ao medo de contaminação por Sars-CoV-2.

Apesar do grande esforço que os profissionais de Educação Física têm realizado para manterem os alunos fisicamente ativos por meio de vídeo-aulas [1,2], a aderência desses à essa nova possibilidade de se exercitar não está sendo unânime. Finalmente, aliados a maior propensão à alta ingestão calórica durante o isolamento social [3], tais fatores contribuem para alavancar o sobrepeso e a obesidade, outro grave problema de saúde pública que afeta a população mundial em diferentes faixas etárias [46].

Adicionalmente aos prejuízos metabólicos e na qualidade de vida ocasionados pelo aumento nos percentuais de gordura corporal, durante o isolamento social, é provável que o descontentamento com a estética corporal, levará inúmeros adultos a buscarem as academias e locais destinados à prática de atividade física, assim que estes locais voltarem a funcionar. Apesar disso, antes mesmo do Profissional de Educação Física abordar

\footnotetext{
${ }^{1}$ Centro Universitário Carlos Drummond de Andrade (Unidrummond). São Paulo, SP, Brasil. Email: erivelton.fernandes@hotmail.com

${ }^{2}$ Universidade de Mogi das Cruzes.

${ }^{3}$ Pós-graduado em Fisiologia do Exercício e Prescrição do Treinamento

${ }^{4}$ Universidade de São Paulo (USP). São Paulo, SP, Brasil.
}

França EF, Macedo MM, de França TR, Cossote DF, Gonçalves L, dos Santos PAC, Miyake GM. Triagem de saúde para participação nos programas de Exercício Físico pós-pandemia de COVID-19: Uma ação necessária e emergente ao Profissional de Educação Física. InterAm J Med Health 2020;3:e202003050. 
os parâmetros necessários para a determinação da zona alvo de treino, até mesmo para os indivíduos fisicamente ativos e familiarizados com a prática de exercícios físicos sistematizados, é necessário ser cauteloso. Isso se faz necessário, sobretudo, na atual conjuntura, em que muitos indivíduos estão há meses sem realizar atividades físicas em intensidade moderada a vigorosa. Sendo assim, este breve artigo tem como objetivo abordar 2 questionário de triagem de saúde para participação nos programas de atividades físicas/condicionamento físico, recomendados pelo American College of Sports Medicine que serão essenciais antes da prescricão de exercicios fisicos pelo essenciais antes da prescriçao de extrios fisicos pelo Profissional de Educaçao Hisica, com a reabertura das academias e locais destinados à prescrição de exercícios fisicos.

O Physical Activity Readiness Questionnaire (PAR-Q), em português, Questionário de Prontidão para Atividade Física, foi desenvolvido na década de 1970 pelo Ministério da Saúde da província canadense de British Columbia endossado pelo Fitness Canada e usado em coniunto com o Canadian Home Fitness Test e o Canadian Standard Test of Fitness [7,8]. Sua utilização não se limitou apenas ao Canadá, passando a ser adotado em vários países, onde

Quadro 1- Questionário de Prontidão para Atividade Física (PAR-Q)

\section{Questionário de Prontidão para Atividade Física (PAR-Q)}

Este questionário tem o objetivo de identificar a necessidade de avaliação por um médico antes do ińcio da atividade física. Caso você responda "SIM" a uma ou mais perguntas, converse com seu médico ANTES de aumentar seu nível atual de atividade física. Mencione este questionário e as perguntas às quais você respondeu "SIM".

Por favor, assinale "SIM" ou "NÃO" às seguintes perguntas:

1. Algum médico já disse que você possui algum problema de coração e que só deveria realizar atividade física supervisionada por profissionais de saúde?

$\square \operatorname{Sim} \square$ Não

2. Você sente dores no peito quando pratica atividade física?

$\square \operatorname{sim}$

口Não

3. No último mês, você sentiu dores no peito quando praticou atividade física?

$\square \operatorname{Sim} \quad \square$ Não

4. Você apresenta desequilíbrio devido à tontura e/ ou perda de consciência?

$\square \operatorname{sim}$

$\square$ Não

viabilidade e importância na identificação para frequentar os estabelecimentos

- para os interessados com idade entre 15 e 69 nos, da resposta ao Questionário de Prontidão para Aidade Física (PAR-Q) que consta do Anexo I desta lei;

Parágrafo único - Dos interessados com idade entre

Composto por sete questões (Quadro 1), caso responda "SIM" a uma ou mais perguntas do PAR-Q, imprescindível que $\mathrm{O}$ indivíduo converse com seu dico antes de iniciar o programa de exercícios físicos tendido.

Termo de Responsabilidade para Prática de Atividade Fisca

Estou ciente de que é recomendável conversar com um médico antes de aumentar meu nível atual de atividade física, por ter respondido "SIM" a uma ou mais perguntas do "Questionário de Prontidão para Atividade Física" (PAR-Q). Assumo plena responsabilidade por qualquer atividade física praticada sem o atendimento a essa recomendação.

Nome completo:
Idade:_L Local:_ Data:____

Adaptado das Diretrizes do ACSM para os Testes de Esforço e sua Prescrição [9]

Outro instrumento que pode ser utilizado com o intuito de verificar fatores de risco durante a prática dos exercícios físicos é o Questionário de Triagem Préparticipação das Instituições de Saúde/Condicionamento Físico da American Heart Association/ACSM [9] Quando Q que estão divididas nos 4 domínios do questionário (histórico, sintomas, fatores de risco cardiovascular e outros problemas de saúde) (Quadro 2) há uma maior precisão na determinação de fatores de risco para a prática segura de exerććcios físicos, sobretudo em relação aos fatores de risco para Doenças Cardiovasculares (DCV) [9]. 
Quadro 1- Questionário de Triagem Pré-participação das Instituiçōes de Saúde/Condicionamento Físico da American Heart Association/ACSM.

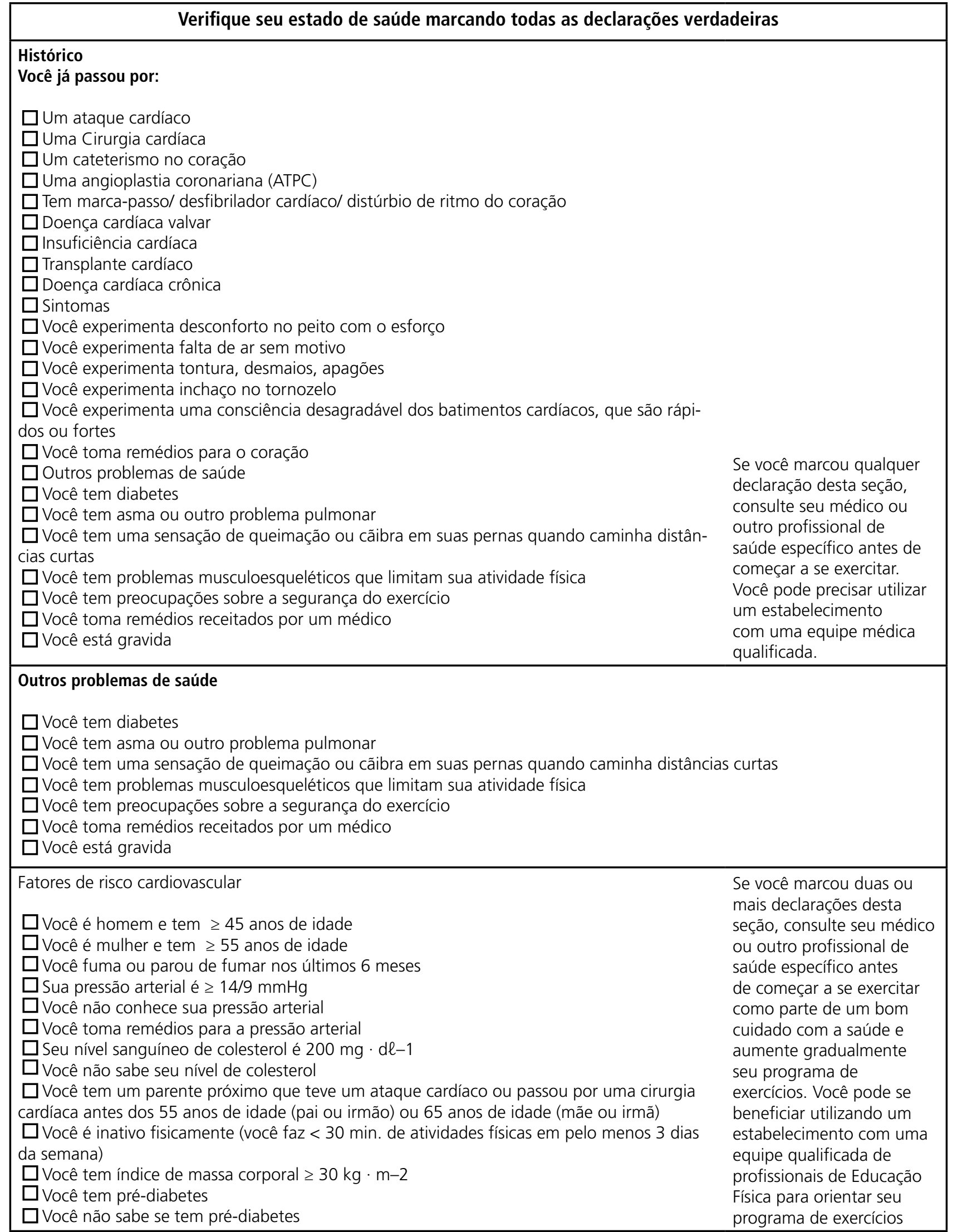

Você deve ser capaz de se exercitar com segurança sem consultar seu medico ou outro profissional de cuidado com a saúde em um programa autoorientado ou em qualquer estabelecimento que satisfaça as suas necessidades de exercício

Retirado das Diretrizes do ACSM para os Testes de Esforço e sua Prescrição [9]

Cabe ressaltar que se durante o preenchimento deste questionário não houver informações suficientes sobre a presença ou a ausência de um fator de risco cardiovascular em específico, este deve ser considerado como presente, exceto o pré-diabetes. Se o critério de pré-diabetes estiver faltando ou for desconhecido, deve ser contado como fator de risco para aqueles $>45$ anos de idade, especialmente com Indice de Massa Corpora IMC $\geq 25 \mathrm{~kg} \cdot \mathrm{m}-2$ e fatores de isco adicionas de DCV para pre para pré-diabetes. Diante disso, a quantidade de fatores de risco positivo é então somada. Adicionalmente, para indivíduos com HDL $\geq 60 \mathrm{mg} \bullet \mathrm{d} \ell-1(1,55 \mathrm{mmol} \bullet \ell-1)$

um fator de risco positivo é subtraído da soma dos fatores positivos. [9].

A avaliac̃ão dos fatores de risco para DCV juntamente com a determinação de doenças pulmonares, renais, metabólicas e cardiovasculares, conforme demonstrado na figura 1, é de suma importância e possibilita ao Profissional de Educação Física obter informações valiosas para a prescrição de exercícios físicos, tais como:

1. Necessidade de avaliação clínica e liberação médica para o aluno/cliente iniciar a prática de exercícios físicos;

2. Obrigatoriedade ou recomendação da realização de teste de esforço antes da prescrição de exercícios físicos; 3. O nível de supervisão durante o teste de esforço e para a participação em programas de exercício físico. 


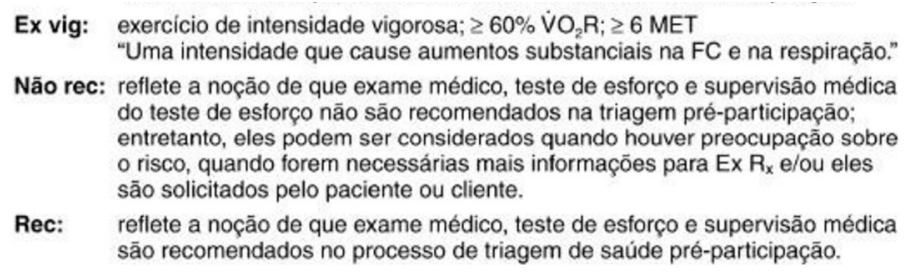

Como é possível observar na figura anterior, indivíduos assintomáticos e com baixo risco de DCV, < 2 fatores de risco (FR), não necessitam de uma avaliação médica clínica para iniciar um programa de exercícios físicos em intensidade moderada a vigorosa, bem como para se exercitar nestas intensidades não necessitam realizar um teste de esforço ou ter a presença de um médico durante a execução do mesmo. Para os indivíduos assintomáticos com risco moderado de $D C V, \geq 2 F R$, se aplicam as mesmas orientações, com uma exceção apenas, que se trata da necessidade de realizar avaliação médica antes de iniciar a prática de exercícios físicos em intensidade vigorosa. Por fim, em indivíduos sintomáticos e com alto risco de DCV para intensidades moderadas e vigorosas de exercícios físicos, é necessário a avaliação clínica prévia, a realização de teste de esforço com supervisão médica, de modo a subsidiar a prescrição de exercícios físicos por um Profissional de Educação Física [9].

\section{REFERÊNCIAS}

1. França, E. F., Miyake, G. M., da Silva Júnior, J. P., Matsudo, V. K. R., Martins, R. Á. L., \& Nascimento, F. D. (2020). COVID-19: Estratégias para se manter fisicamente ativo e seguro dentro de casa. InterAmerican Journal of Medicine and Health, 3, 1-10.

2. Miguel, H. (2020). Impactos da Covid-19 sobre o Personal Trainer. InterAmerican Journal of Medicine and Health, 3.

3. Muscogiuri, G., Barrea, L., Savastano, S., \& Colao, A. (2020). Nutritional recommendations for CoVID-19 quarantine. European Journal of Clinical Nutrition, 1-2.

4. Abarca-Gómez, L., Abdeen, Z. A., Hamid, Z. A., AbuRmeileh, N. M., Acosta-Cazares, B., Acuin, C., ... \& Agyemang, C. (2017). Worldwide trends in body-mass index, underweight, overweight, and obesity from 1975 to 2016: a pooled analysis of 2416 population-based measurementstudies in 128.9 million children, adolescents, and adults. The Lancet, 390(10113), 2627-2642.

5. FRANÇA, Erivelton Fernandes et al. 2020, The Influence of Excessive Screen Time on the Level of Physical Activity, Excess Weight and Back Pain in Childhood: A Literature Review. Int J Recent Sci Res. 11(03), pp. 37822-37827.

6. FRANÇA, Erivelton Fernandes et al. 2020, Physical activity level and nutritional status associated with screen habits of students living in Brazil. International journal of physical education, sports and health, 7 (03) pp. 248-253.

7. Adams, R. (1999). Revised Physical Activity Readiness Questionnaire. Canadian Family Physician, 45, 992.

8. ChisholmDM, Collis ML, KulakLL, DavenportW, GruberN. (1975)Physicalactivityreadiness. BrColMedJ,17pp.375-78.

9. American College of Sports Medicine. Diretrizes do ACSM para os Testes de Esforço e sua Prescrição. 10a edição. Rio de Janeiro: Guanabara Koogan, 2018.

10. Brasil. Lei n.16.724, 22 de maio de 2018. Acrescenta 0 artigo $5^{\circ}$-A, altera o artigo $6^{\circ}$ e revoga o "caput" do artigo $5^{\circ}$ da Lei $n^{\circ} 10.848$, de 06 de julho de 2001. Assembléia Legislativa do Estado de São Paulo (SP). 